\title{
The Vegetation Extraction and Hierarchical Classification using an IRS-1C LISS III Image
}

\author{
Rubina Parveen \\ Research Scholar \\ VTU, Belagavi \\ Karnataka, India
}

\author{
Subhash Kulkarni \\ PESIT (South Campus) \\ Bangaluru \\ Karnataka, India
}

\author{
V. D. Mytri \\ AIET \\ Kalaburagi \\ Karnataka, India
}

\begin{abstract}
Extraction of vegetation is an important step for agricultural, forest and greenery mapping. The proposed method examines the complex process of land cover vegetation pattern classification using an IRS-1C LISS III image. Pre-processing was done by employing partial differential equation (PDE). Normalized differential vegetation index (NDVI) was applied to separate vegetation features from the image. Agricultural and non-agricultural vegetation features were the major and divergent hierarchical trends, which were observed. Further, classification was done by generating grey Level Co-occurrence Matrix (GLCM). Goal of this paper was to explore vegetation patterns by masking other features and identification of different vegetation patterns. Firstly, area of different land covered features was calculated. Then vegetation occupancy was calculated. finally, hierarchal separation of vegetation types was done to extract various vegetation patterns. Further, ground truth verification was done by Google Earth Images of same period, of relatively same area. From the results, it was demonstrated that various vegetation patterns were extracted, accurately and automatically.
\end{abstract}

\section{General Terms}

IRS-1C LISS III Image, classification

\section{Keywords}

Partial-Differential Equation (PDE), Normalized differential vegetation index (NDVI), Level set method, Grey Level Co-occurrence Matrix (GLCM)

\section{INTRODUCTION}

Remote Sensing Images are very complex and information extraction is a very challenging task for analysis. One of the greatest advantages of satellite imaging is land use analysis by generation of information in spatial and temporal domain. Successful analysis of these images, interpretation and validation [1] depends on the clarity of the data and analysis technique used. Images can be influenced by many external and internal factors [2] like atmosphere and sensors quality, etc. Generation of information about vegetation occupancy, temporal changes, deforestation and land use for vegetation has to be monitored continuously. Existing methods measures vegetation in two ways; field measurements and remote sensing techniques [3]. Field measurements are time consuming and less efficient while, high temporal and spatial resolution satellite images can provide more accurate feature extraction [4]. Identification of geographical features from low resolution multi-spectral scanner (MSS) image is a vital task. Spectral reflectance characteristics vary under different environments $[5]$ and time periods. A linear image self-scanning sensor (LISS - III) used in IRS-1C, is a multi-spectral camera operating with spectral combinations 0.52 - 0.59 (Green), 0.62 - 0.68 (Red), 0.77 - 0.86 (Near Infra-red) and 1.55 - 1.70 (Mid Infra-red) [5], [6]. A clear understanding of spectral reflectance characteristics is a prerequisite for interpreting distinctive features in an MSS image. The vegetation distributes very sparsely at some areas and densely at some other areas, in the study image. As satellite sensor resolution is not high enough, the remote sensing image here is expressed by mixed pixels [7] with presence of two or more feature in single pixel. If the window size is too small, insufficient ground level information is extracted to characterize a specific land cover and if the window size is too large, it can overlap two types of ground cover or mixed pixels will occur and thus introduce erroneous spatial information [8]. So frames of satellite image were divided into an average area which was expedient for analysis. Level set was a curve propagation algorithm based on the PDE based method, this provides a direct way to estimate the geometric properties of the evolving structure [2]. Potential application of LISS III data is Crop discrimination and vegetation Dynamics [9] . Investigation was done for efficient segmentation of vegetation into different vegetation patterns and to build a taxonomic classification partitions of observation space into the disjoint region [10]. Vegetation was further divided into hierarchical clusters of vegetation types. Qualitative analysis was done visually and Classification was followed by area calculation, as the last course of action, for quantitative analysis. For the given IRS-1C LISS III image, the main objectives were to:

(1) Explore the efficiency of level set segmentation technique by applying PDEs based pre-processing.

(2) Demarcation of administrative boundaries and to prepare vegetation cover maps from given satellite imageries .

(3) Hierarchical classification of vegetation to get different vegetation patterns Classification with greater degree of accuracy.

The succeeding section throws light on study area and the geographic features associated with it. 


\section{STUDY AREA DETAILS}

Study area taken is a part of Yadgir District area, which is highly heterogeneous. Yadgir is the district of Karnataka state, India. Agriculture in the district mainly depends upon the rainfall and the net area irrigated is 14 percentage in the district. The zones indicate the predominance of rain dependent and dry land agricultural area [11]. The area taken for the study is mainly covered with water body, irrigated land, rain fed land and sparse vegetation.

\subsection{Earth Observation}

The study area is between the longitude $162649.04 * 94 \mathrm{~N}$ to and latitude $765002.96 * 94 \mathrm{E}$. This is an IRS-1C LISS III data acquired in 20th October 2006 with a spatial resolution 23.5mts. Data obtained by Google Earth was used as ground truth information for the classification of the images. The Fig. 11 shows the area of analysis.

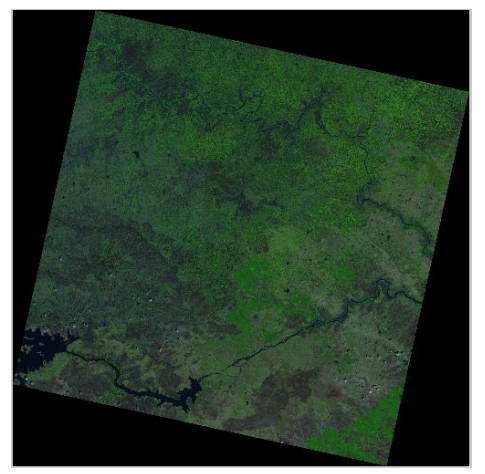

Fig. 1. IRS-1C LISS III image acquired on 20th October 2006

Simulation was performed using Matlab (R2012a) and ERDAS Imagine 9.1. Data pre-processing, image segmentation, classification and computation is done using the tools supported by MATLAB. The study image was classified into broad and detailed land use classes, by unsupervised classification. Classification was done into probable five classes as per the geo-topology of the study area. Resulting image was shown in Fig. 2

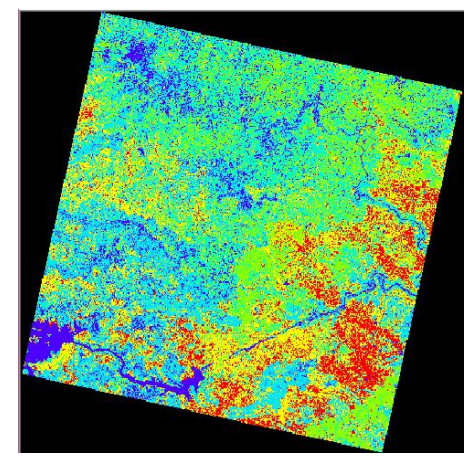

Fig. 2. Unsupervised Classification Image

Histogram, color of land cover features and relative class names along with coverage area was tabulated in Table 1 From visual interpretation and Google Earth image of same area and same time, green color in the Fig. 2 depicts vegetation land cover. The area of vegetation coverage is 548458 Hectare.

\section{METHODOLOGY}

The algorithm has been divided into two steps. First, extraction of vegetation and second, hierarchical classification of extracted vegetation.

\subsection{Flow Chart}

Initially, the band with high spectral reflectance for vegetation were taken and stacked together and one RGB image was formed [6]. In an image (LISS III) taken from IRS satellite, a pixel covers an area of approx. 552.2 sq. $\mathrm{m}$ or 0.0552 hectare [5]. Given Image was read in MATLAB $(7069 * 7052 * 3$ pixels). Due to computational complexity and visual clarity of the data, given frame was divided into 16 expedient windows. A block size of 589*587 pixels would thus contain a significant amount of territorial information. The proposed scheme is shown using block diagram in Fig. 3 Proposed work has been divided onto four levels as shown in Fig. 4

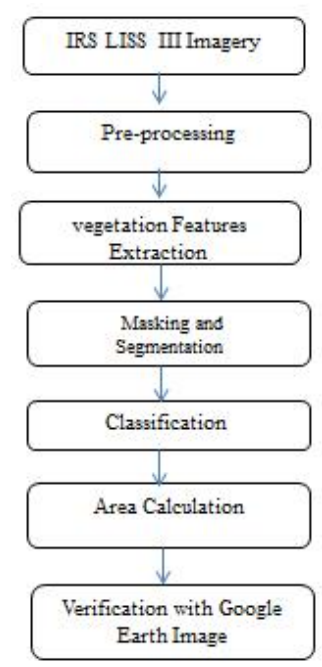

Fig. 3. Flow diagram for hierarchical classification

Level set based hierarchical clustering was used for segmentation of the image. Level 1: Land Base- The study area mainly includes features like water body, un-cultivated open lands and settlements, vegetation, sandy Soil and barren lands/fallow land, broadly vegetation and non-vegetation areas. Level 2: Land cover type-This level divides vegetation into tree and non-trees type of vegetation. Level 3: Vegetation Type: Concentration of the study was hierarchical classification of vegetation, so study area was divided into Systematic agricultural patters and sparse non-agricultural shrubs. Level 4: Density classes: Depending on the density of spread of vegetation was further divided into irrigated land, rain fed land and sparse shrubs.
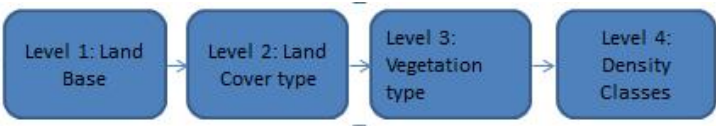

Fig. 4. Hierarchical classification of Study Area 
Table 1. Statistics of Unsupervised Classification.

\begin{tabular}{|l|l|l|l|r|}
\hline Sl no & Histogram & Color & Classes & Area in Hector \\
\hline 1 & 3423015 & Blue & Water Bodies & 273220 \\
\hline 2 & 8105960 & Cyan & Uncultivated open lands and settlements & 485577 \\
\hline 3 & 6978245 & Yellow & Barren Lands & 440579 \\
\hline 4 & 6024977 & Green & Vegetation & 548458 \\
\hline 5 & 3126153 & Red & Sandy Lands & 274387 \\
\hline
\end{tabular}

\subsection{Pre-Processing}

Geometrically registered images were pre-processed by applying partial differential equations (PDEs) filters [12] as an enhancement process. The method employs the similarity between the different band images in a multicomponent image [13]. Most of the PDE driven signal processing techniques were slow, results are good [2] . IRS-1C LISS III image is multi-spectral image. Noise free image band was used as priors in enhancement process. Auxiliary image from another sensor is introduced as reference image or base image into partial differential equation in enhancement process. Similarity of the directions of the edges and correlation between the auxiliary image and the base image is used to smooth out more noise and conserve more detail. Resulting image was subjected to histogram equalization. This preserves the brightness and produces more natural looking image. PDE based enhanced image and the histogram equalized image was shown below in Fig 5 a and b clearly demonstrates the visual clarity as compared to original image. The first objective of the study was attained; the results are visually vibrant than the original frame. Temporal and spectral signature play vi-

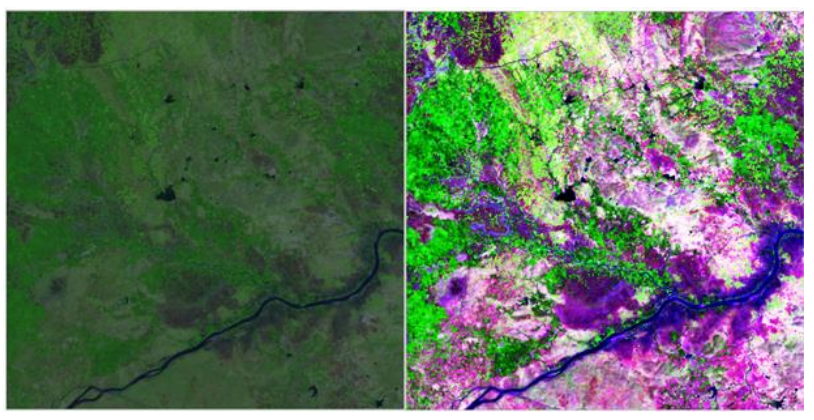

Fig. 5. a. Enhanced image and b. Histogram Equalized image.

tal role in segmentation [14]. Color is an immediately perceivable visual feature [5]. The land use classes in the study area include built up areas, barren lands, vegetation and water bodies etc. As the research objective is to extract vegetation patterns, Normalized Difference Vegetation Index (NDVI) [15] was applied for discriminating vegetation from non- vegetation features. The lower the red values reflectance, the higher is the chlorophyll content [16] in the biomass [17]. NDVI was defined by an algebraic formula. 1]

$$
N D V I=\frac{N I R-R E D}{N I R+R E D}
$$

Where, NIR: Near Infrared Band and R: Red band. A relation between these two bands allows calculating indices of vegetation. NDVI for a given pixel always results in a number that ranges from -1 to +1 . Generally, non-vegetated areas gives values close to zero and vegetated areas gives values close to one indicating the high possible density of green leaves [8]. The NDVI images were examined, mean and standard deviation values were calculated and a thresholding technique was applied to separate vegetation from other land cover. The intersection of original image with NDVI images produces a vegetation mask image [18]. Ignoring significant areas and capturing the texture property of vegetation only, results in the image shown in Fig. 6 . As the research was focused on vege-

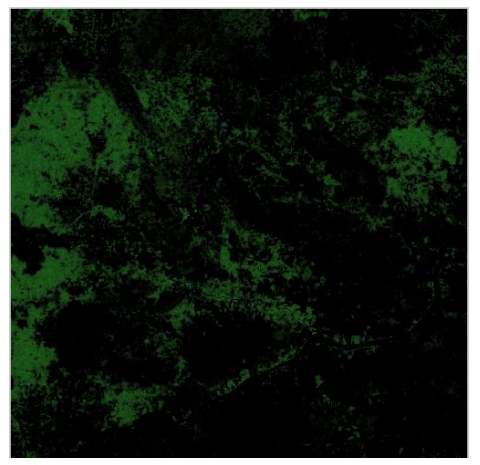

Fig. 6. Vegetation Extraction

tation pattern analysis, vegetation was extracted and other features areas masked. Further, for segmentation level set method was used. Level set method was an active contour based model. The method iterates over the image creating estimate of the density at each pixel and links each pixel to the nearest pixel, increasing the estimate of the density [2]. The method uses an active curve objective functional with two terms: an original term which evaluates the deviation of the mapped image data within each segmentation region from the piecewise constant model and a classic length regularization term for smooth region boundaries [19], [20]. The image was subjected to 500 iterations for appropriate segmentation. By this second objective was achieved and resulting Fig. 6has only vegetation features.

\subsection{Classification}

Signature analysis may involve developing Grey Level Cooccurrences Matrix (GLCM) analyses of the mean, variances and covariance [21].In GLCM, the number of occurrences of the pair of grey levels $i$ and $j$ which were at a distance $d$ apart in original image corresponds to each entry $I(i, j)[22]$. Parameters are defined based on GLCM and were computed by MATLAB, as shown in Table. 2 . Statistical features were used to estimate the similarity between different grey level co-occurrence matrices. To create feature database such as mean was calculated form original image and a set of features such as variance, energy and entropy were calculated from the co-occurrence matrix $\mathrm{C}(\mathrm{i}, \mathrm{j})$ using the formulas given in equation. 2 to equation. 5 , shown below.

$$
\text { mean, } \mu=\mu_{1}=\sum x P(x)
$$




$$
\text { variance, } \sigma^{2}=\mu_{2}=\sum\left(x-\mu_{1}\right)^{2} P(x)
$$

$$
\text { variance, } E=\sum c(i, j)^{2}
$$

$$
\text { Entropy }=-\sum \sum C(i, j) \log C(i, j)^{2}
$$

These features were stored in the feature library, which were further used for classification as shown in the Table 2 Pixel with value i occurs horizontally adjacent to a pixel with the value j. Each element $(i, j)$ in Co-occurrence matrix specifies the number of times that the pixel with value $\mathrm{i}$ occurs horizontally adjacent to a pixel with the value $\mathrm{j}[\overline{8}]$. Using total number of pixels in an image and

Table 2. GLCM Features

\begin{tabular}{|l|l|l|c|c|}
\hline Feature & Grass & Agricultural land & Shrubs & Small Trees \\
\hline Mean & 0.01 & 0.02 & 9.7 & 12.27 \\
\hline Variance & 0.01 & 4.71 & 124.91 & 227.06 \\
\hline Energy & 1.86 & 0.37 & 0.72 & 0.71 \\
\hline Entropy & 0.50 & 5.09 & 3.53 & 3.41 \\
\hline
\end{tabular}

pixels present in each clustered image, percentage of each cluster in an image has been computed. These percentages along with the total area under image have been used to calculate area under each cluster [22]. The classified results were shown in Fig. 7 ] Color differencing was used to identify each pixel with different reflectance. The classification quality of vegetation depends strongly

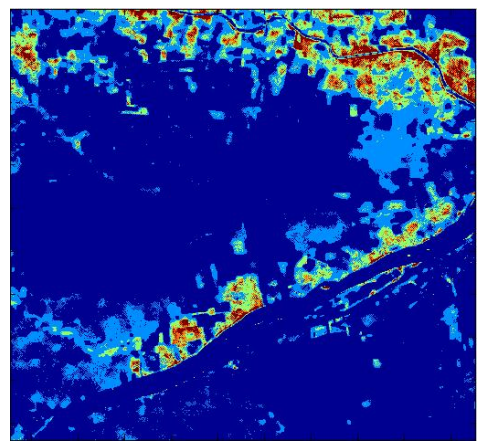

Fig. 7. Vegetation Segmented Image

on the level of the hierarchy [16]. Spectral Euclidean distance was used to define the vegetation signatures. The image (as shown in Fig. 7] [22]. From the Fig. 7] sky blue color depicts Grass, brown depicts agricultural crops, green depicts small shrubs and orange color depicts Small trees and Dark blue depicts masked area.

\section{RESULTS AND DISCUSSION}

A specific variation in the reflectivity of vegetation depicts a variety of vegetation. These spectral properties were explored to get hierarchical classification. Results of the discussed strategy were shown in the succeeding images. Field verification has been done by comparing the results with Google Earth image. The work was done by visual image interpretation [23|. Crop fields to be extracted are defined with clear spatial crop pattern [24] were shown in Fig. 8 Brown colored pixels from the segmented image are extracted.
It was observed from the results that most of the land in the study area is irrigated and very ample amount of land was rain fed, as the image window was very close to river stream. The captured image

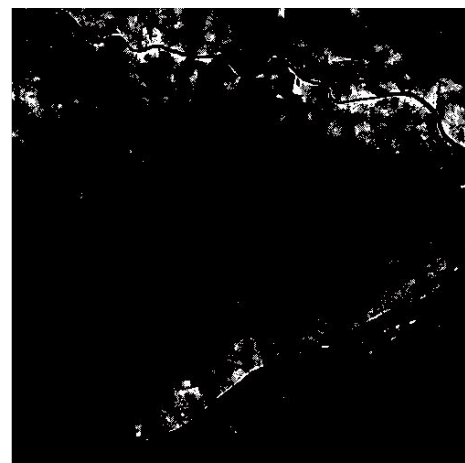

Fig. 8. Agricultural Land patterns

is of autumn season, Sky blue color from the Fig. 7 depicts grass. More area was covered by grass as shown in Fig. 9 due to seasonal appearances. Boundaries of the fields are surrounded by or-

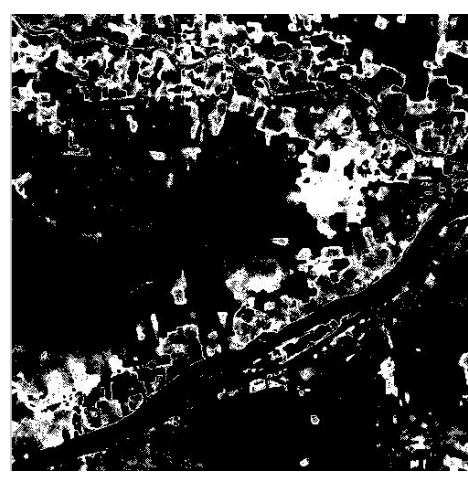

Fig. 9. Grass areas

ange color, in the classified image. Orange color pixels (as shown in Fig. 7) depict small trees. Big size of the ground objects were better extracted at high scale parameter while smaller ground objects are better captured using low scale parameter for segmentation [20]. Small trees were the boundaries which are planted to protect crops from farm animals and other interlopers. In this season deciduous trees lose their leaves. The results were analyzed on scale 20 for visible clarity, as shown in Fig. 10 Woody plants smaller than a tree, and usually with several stems from the same base were observed by green color, in Fig. 7. These shrubs may be weeds, which are usually found surrounding the field. This was shown in Fig. reffig:smallShrubs. Most of the area in the classified image was dark blue in color which depicts other part of the image like water bodies, fallow land and Settlements, etc. Google Earth image of 31st March 2006 was referred to prove the correctness of the results. Similar patterns of vegetation of agricultural land, grass areas, small trees and small shrubs were observed from Google Earth Image. After classification of an image, area under each cluster was calculated. For that purpose, the number of pixels in each class has been calculated to calculate individual class area |22]. Using total number of pixels in an image and pixels present in each classified image, area and percentage occupancy of particular land cover is 


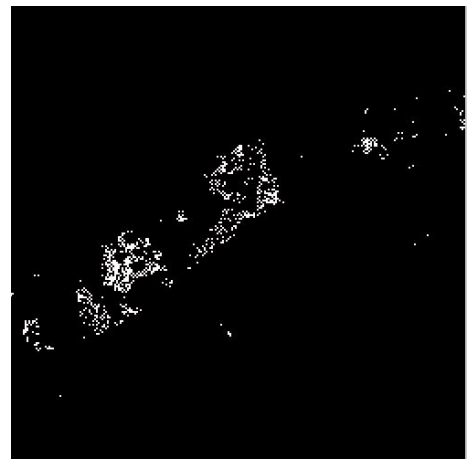

Fig. 10. Small Trees

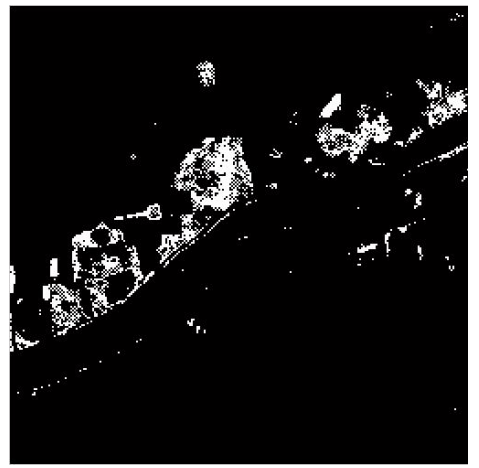

Fig. 11. Small Shrubs

calculated, shown in Table 3. Grasslands cover 16161.5 hectares; Agricultural Land covers 4961.0 hectares, Shrubs covers 9370.4 hectares and Small trees covers 49.64 hectares of area. One out of

\begin{tabular}{|l|l|l|l|}
\hline Classes & $\begin{array}{l}\text { Number of } \\
\text { Pixels }\end{array}$ & $\begin{array}{l}\text { Area in Sq. } \\
\text { mts }\end{array}$ & $\begin{array}{l}\text { Area in } \\
\text { Hectares }\end{array}$ \\
\hline $\begin{array}{l}\text { Small } \\
\text { Trees }\end{array}$ & 899 & 496472.75 & 49.647275 \\
\hline $\begin{array}{l}\text { Agricult } \\
\text { ural } \\
\text { Land }\end{array}$ & 89834 & 49610826.5 & 4961.08265 \\
\hline Shrubs & 169677 & 93704123.25 & 9370.412325 \\
\hline Grass & 292649 & 161615410.3 & 16161.54103 \\
\hline Total & 553059.00 & 305426832.75 & 30542.68 \\
\hline Approx. area of 16 windows & 488682.9324 \\
\hline $\begin{array}{l}\text { Area of vegetation by unsupervised } \\
\text { classification }\end{array}$ & 548459 \\
\hline \multicolumn{4}{|l|}{} \\
\hline \multicolumn{4}{|l|}{ Percentage of accuracy } \\
\hline
\end{tabular}

Fig. 12. Table III. Hierarchical Classification statistics.

16 windows covers 30542.68 hectares of total vegetation area. Vegetation pattern in each window may vary due to random blowout. Area covered by 16 windows is approximately 488682.9 hectares. Unsupervised classification (shown in Table. 1) has resulted in total vegetation cover of 548549 Hectares. Comparing the results the third objective of the study i.e. hierarchical classification of vegetation patterns, was also achieved with 89.10 Percentage of accuracy. All four classes vary in percentage of land cover. Graph in Fig. 13 clearly shows dissimilarity in percentage of land cover in the study

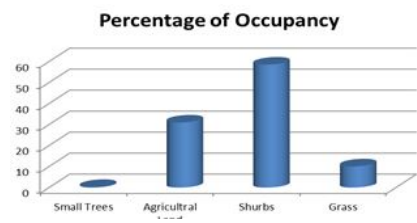

Fig. 13. Percentage of vegetation classes Occupancy

window. Small trees were very less in number i.e. 0.31 percentage while agricultural lands were having 31 percentage of occupancy. Similarly, Shrubs such as weeds have largely occupied the land cover i.e. 58 percentage with only 10 percentage of grass occupancy.

\section{CONCLUSION}

Remote sensing the data is too large, expensive, and analysis accuracy depends on too many parameters. This algorithm highlights vegetation land cover and will inevitably weaken non vegetation land cover. This study has been made to develop a generalized algorithm to extract all vegetation features automatically. PDE based enhancement with level set segmentation gives apparently more clear results. Signature sets have been developed for vegetation land cover, and based on these results four class of vegetation patters were identified. Attempt has been made to develop an automatic hierarchical classification of land cover vegetation pattern using IRS-1C LISS III image, by comparing the results with Google Earth Image as ground truth verification. This experimentation will help to identify agricultural land covers and non-agricultural land covers, without much efforts of the physical survey. This hepls user to identify various patters of vegetation, which can be used as the base for crop identification and discrimination of health of vegetation. Handling complete frames at a time, difficulties of massive data processing caused by segmentation and calculation of characteristic parameters is yet to be revisited. The algorithm can be stretched for multi-temporal images and its relative change detection.

\section{REFERENCES}

[1] A. Raj and N. Vijayan, "Analysis of landuse landcover changes of kazhakuttam block based on gis," in Green Technologies (ICGT), 2012 International Conference on, pp. 143146, IEEE, 2012.

[2] S. Bharathi, V. Shreyas, R. Anirudh, S. Sanketh, P. D. Shenoy, K. Venugopal, and L. Patnaik, "Performance analysis of segmentation techniques for land cover types using remote sensing images," in 2012 Annual IEEE India Conference (INDICON), pp. 775-780, IEEE, 2012.

[3] M. Kırcı, E. O. Güneş, Y. Çakır, and S. Şentiirk, "Vegetation measurement using image processing methods," in Agrogeoinformatics (Agro-geoinformatics 2014), Third International Conference on, pp. 1-5, IEEE, 2014.

[4] D. Chakraborty, G. K. Sen, and S. Hazra, "High-resolution satellite image segmentation using hölder exponents," Journal of Earth System Science, vol. 118, no. 5, pp. 609-617, 2009.

[5] D. Verma, N. Garg, N. Garg, N. Mishra, and G. Dosi, "Development of descriptors for natural feature identification on irs liss iii images," in Methods and Models in Computer Science (ICM2CS), 2010 International Conference on, pp. 5458, IEEE, 2010. 
[6] A. Upadhyay and S. K. Singh, "Classification of irs liss-iii images using pnn," in Computing for Sustainable Global Development (INDIACom), 2015 2nd International Conference on, pp. 416-420, IEEE, 2015.

[7] H.-b. Zhao, T. Liu, Y.-p. Cui, and J.-q. Lei, "Using multispectral remote sensing data to extract and analyze the vegetation information in desert areas," in Environmental Science and Information Application Technology, 2009. ESIAT 2009. International Conference on, vol. 3, pp. 697-702, IEEE, 2009.

[8] S. Rajesh and S. Arivazhagan, "Land cover/land use mapping using different wavelet packet transforms for liss iv imagery," in Computer, Communication and Electrical Technology (ICCCET), 2011 International Conference on, pp. 103108, IEEE, 2011.

[9] N. Gautam, "Irs-1c applications for land use/land cover mapping, change detection and planning," in Geoscience and Remote Sensing, 1997. IGARSS'97. Remote Sensing-A Scientific Vision for Sustainable Development., 1997 IEEE International, vol. 4, pp. 1775-1777, IEEE, 1997.

[10] Y. Xie, Z. Sha, and M. Yu, "Remote sensing imagery in vegetation mapping: a review," Journal of plant ecology, vol. 1, no. 1, pp. 9-23, 2008.

[11] N. Gautam, "Geographical features and socio-economic and cultural characteristic of yadgir district," in Online Database, GOK.

[12] F. Mirzapour and H. Ghassemian, "Hyperspectral image classification using profiles based on partial differential equations," in 2015 23rd Iranian Conference on Electrical Engineering, pp. 288-292, IEEE, 2015.

[13] P. Liu, F. Huang, G. Li, and Z. Liu, "Remote-sensing image denoising using partial differential equations and auxiliary images as priors," IEEE Geoscience and Remote Sensing Letters, vol. 9, no. 3, pp. 358-362, 2012.

[14] H. North, D. Pairman, S. E. Belliss, and J. Cuff, "Classifying agricultural land uses with time series of satellite images," in 2012 IEEE International Geoscience and Remote Sensing Symposium, pp. 5693-5696, IEEE, 2012.

[15] A. Mermer, H. Yıldız, E. Ünal, M. Aydoğdu, A. Özaydın, F. Dedeoğlu, O. Urla, O. ydoğmuş, H. Torunlar, M. Tuğaç, et al., "Monitoring rangeland vegetation through time series satellite images (ndvi) in central anatolia region," in AgroGeoinformatics (Agro-geoinformatics), 2015 Fourth International Conference on, pp. 213-216, IEEE, 2015.

[16] G. Ons and R. Tebourbi, "Object oriented hierarchical classification of high resolution remote sensing images," in 2009 16th IEEE International Conference on Image Processing (ICIP), pp. 1681-1684, IEEE, 2009.

[17] B. Adriano, H. Gokon, E. Mas, S. Koshimura, W. Liu, and M. Matsuoka, "Extraction of damaged areas due to the 2013 haiyan typhoon using aster data," in 2014 IEEE Geoscience and Remote Sensing Symposium, pp. 2154-2157, IEEE, 2014.

[18] M. Bandyopadhyay, J. A. van Aardt, and K. CawseNicholson, "Classification and extraction of trees and buildings from urban scenes using discrete return lidar and aerial color imagery," in SPIE Defense, Security, and Sensing, pp. 873105-873105, International Society for Optics and Photonics, 2013.

[19] M. B. Salah, A. Mitiche, and I. B. Ayed, "Effective level set image segmentation with a kernel induced data term," IEEE
Transactions on Image Processing, vol. 19, no. 1, pp. 220232, 2010.

[20] P. Garg et al., "Texture based information extraction from high resolution images using object based classification approach," in Earth Observation and Remote Sensing Applications (EORSA), 2014 3rd International Workshop on, pp. 299-303, IEEE, 2014.

[21] P. S. Bharatkar and R. Patel, "Evaluation of rsi classification methods for effective land use mapping," in Communication Systems and Network Technologies (CSNT), 2013 International Conference on, pp. 109-113, IEEE, 2013.

[22] M. Aher, S. Pradhan, and Y. Dandawate, "Rainfall estimation over roof-top using land-cover classification of google earth images," in Electronic Systems, Signal Processing and Computing Technologies (ICESC), 2014 International Conference on, pp. 111-116, IEEE, 2014.

[23] P. K. Rai, S. Gupta, A. Mishra, and M. Onagh, "Multiseasonal irs-1c liss iii satellite data for change detection analysis and accuracy assessement: A case study," Journal of GIS Trends, vol. 2, no. 1, pp. 13-19, 2011.

[24] J. Susaki and R. Shibasaki, "Crop field extraction method based on texture analysis and automatic threshold determination," in Geoscience and Remote Sensing Symposium, 1999. IGARSS'99 Proceedings. IEEE 1999 International, vol. 2, pp. 773-775, IEEE, 1999. 